\title{
Effect of micro-defects on structure failure
}

\section{Coupling asymptotic analysis and strong discontinuity}

\author{
Virginie Bonnaillie-Noël ${ }^{1}$ - Delphine Brancherie ${ }^{2}$ \\ Marc Dambrine $^{3}$ - Sébastien Tordeux ${ }^{4}$ - Grégory Vial ${ }^{5}$ \\ 1) IRMAR - UMR6625, ENS Cachan Bretagne, Univ. Rennes 1, CNRS, UEB \\ av Robert Schuman, F-35170 Bruz \\ virginie.bonnaillie@bretagne.ens-cachan.fr \\ 2) Laboratoire Roberval, Université de Technologie de Compiègne - UMR6253 \\ rue Personne de Roberval, BP 20529, F-60205 Compiègne cedex \\ delphine.brancherie@utc.fr
}

3) LMAP - UMR5142 - Université de Pau et des Pays de l'Adour Avenue de l'Université, BP 1155 F-64013 Pau cedex marc.dambrine@univ-pau.fr

4) Mathematical Institute of Toulouse (Toulouse University, INSA-UMR5219) 135 avenue de Rangueil, F-31077 Toulouse sebastien.tordeux@insa-toulouse.fr

5) IRMAR - UMR6625, ENS Cachan Bretagne, CNRS, UEB av Robert Schuman, F-35170 Bruz

gregory.vial@bretagne.ens-cachan.fr

ABSTRACT. This work aims at taking into account the influence of geometrical defects on the behavior till complete failure of structures. This is achieved without any fine description of the exact geometry of the perturbations. The proposed strategy is based on two approaches: asymptotic analysis of Navier equations and strong discontinuity approach.

RÉSUMÉ. L'objectif de ce travail est de prendre en compte l'influence de la présence de défauts géométriques sur le comportement à rupture des structures et ce, sans description fine de la géométrie particulière des perturbations. L'approche proposée s'appuie sur deux outils : une analyse asymptotique des équations de Navier et l'utilisation de modèles à discontinuité forte. KEYWORDS: multi-scale asymptotic analysis, singular perturbation, strong discontinuity, failure.

MOTS-CLÉS : analyse asymptotique, perturbation singulière, discontinuité forte, rupture.

DOI:10.3166/EJCM.19.165-175 @ 2010 Lavoisier, Paris

EJCM - 19/2010. Giens 2009, pages 165 to 175 


\section{Introduction}

The evaluation of the limit load and behavior till rupture of a structure is highly correlated to the presence of small defects or heterogeneities: material defects, geometrical defects or loading perturbations.

In this work we look more precisely at the prediction of the rupture of complex structures suffering from (surfacic) "small" perturbations (inclusions or porosities). We are concerned with the choice of the most suitable Finite Element strategy to capture the structural behavior. The main feature of our work is to propose an approach dealing with both singular perturbations and localization zones development, by using a coarse description of the geometry: neither the perturbation shape nor a fine representation of the cohesive crack are considered. Our aim is to design a numerical strategy dealing with a coarse discretization of the unperturbed domain and able to perform the analysis of the structural response from the elastic phase to complete failure. To that purpose, we consider two macroscopic models dedicated to each of the two phases of the behavior:

- the asymptotic analysis is used to evaluate the influence of the presence of microdefects on the solution (Dambrine et al., 2005; Bonnaillie-Noël et al., 2009),

- the strong discontinuity approach allows taking into account, at the structural scale, the development of localization zones or cohesive cracks (Brancherie et al., 2009).

In the first section, we give the keypoints of the asymptotic analysis and the numerical strategy used for the evaluation of stress concentration due to the presence of geometrical defects. We present, in the second section, the approach developed to couple the asymptotic analysis to the strong discontinuity method in order to perform the computation of the behavior of the structure till complete failure. Finally, the third section is dedicated to some numerical results obtained considering structures presenting several singular perturbations.

\section{Description of the influence of singular perturbations: multi-scale asymptotic analysis}

We evaluate the influence of geometrical perturbations by a multi-scale asymptotic analysis of Navier equations of linear elasticity.

We consider here a domain $\Omega_{\varepsilon}$ pierced with a perturbation of size $\varepsilon$ centered on the regular point 0 (see Figure 1). In the following, we denote as $\Omega_{0}$ the unperturbed domain and $\mathbf{H}_{\infty}$ the unbounded domain obtained by a blow-up around the point 0 bringing the perturbation at scale $1: \mathbf{H}_{\infty}=\lim _{\varepsilon \rightarrow 0} \Omega_{\varepsilon} / \varepsilon$. 


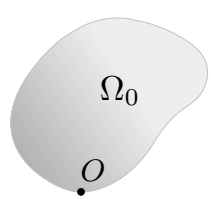

(a)

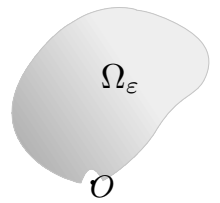

(b)

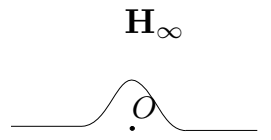

(c)

Figure 1. Unperturbed domain $\Omega_{0}$ (a), perturbed domain $\Omega_{\varepsilon}$ (b) and unbounded do$\operatorname{main} \mathbf{H}_{\infty}$ (c)

The problem we focus on is written on the perturbed domain as:

$$
\left\{\begin{array}{l}
-\mu \Delta \mathbf{u}_{\varepsilon}-(\lambda+\mu) \operatorname{grad} \operatorname{div} \mathbf{u}_{\varepsilon}=\mathbf{f} \text { on } \Omega_{\varepsilon}, \\
\mathbf{u}_{\varepsilon}=\mathbf{u}^{d} \text { on } \Gamma_{d}, \\
\boldsymbol{\sigma} \cdot \mathbf{n}=\mathbf{g} \text { on } \Gamma_{t},
\end{array}\right.
$$

where $\Gamma_{d}$ and $\Gamma_{t}$ denote the Dirichlet and Neumann boundary of the domain respectively, $\Gamma_{t}$ includes the boundary of the perturbation and $\mathbf{g}$ is supposed to be zero in a neighborhood of the perturbation.

We can note that two scales are naturally involved in Problem [1]: the scale of the structure and the scale of the perturbation $\varepsilon$. It has been proven in (Dambrine et al., 2005) that the solution of problem [1] is approximated at first order by the superposition of the solution obtained on the unperturbed domain $\Omega_{0}$ and a correction written in terms of the fast variable $\mathbf{x} / \varepsilon$. We have then:

$$
\mathbf{u}_{\varepsilon}(\mathbf{x}) \simeq \mathbf{u}_{0}(\mathbf{x})-\varepsilon\left[\alpha_{1} \mathbf{V}_{1}\left(\frac{\mathbf{x}}{\varepsilon}\right)+\alpha_{2} \mathbf{V}_{2}\left(\frac{\mathbf{x}}{\varepsilon}\right)\right]
$$

with $\mathbf{u}_{0}$ the solution on the unperturbed domain, $\alpha_{1}=\sigma_{11}\left(\mathbf{u}_{0}\right)(\mathbf{0})$ and $\alpha_{2}=$ $\sigma_{12}\left(\mathbf{u}_{0}\right)(\mathbf{0})$. The profiles $\mathbf{V}_{1}$ et $\mathbf{V}_{2}$ are obtained as solution of an homogeneous Navier equation stated on the unbounded domain $\mathbf{H}_{\infty}$ with Neumann boundary conditions on the boundary of the normalized perturbation $\boldsymbol{\sigma}\left(\mathbf{V}_{\ell}\right) \cdot \mathbf{n}=\mathbf{G}_{\ell}$ (with $\mathbf{G}_{1}=\left(\mathbf{n}_{1}, 0\right)$ and $\mathbf{G}_{2}=\left(0, \mathbf{n}_{1}\right), \mathbf{n}_{1}$ denotes the first component of the outer normal to $\left.\partial \mathbf{H}_{\infty}\right)$ :

$$
\left\{\begin{array}{l}
-\mu \Delta \mathbf{V}_{\ell}-(\lambda+\mu) \operatorname{grad} \operatorname{div} \mathbf{V}_{\ell}=\mathbf{0} \text { in } \mathbf{H}_{\infty} \\
\boldsymbol{\sigma}\left(\mathbf{V}_{\ell}\right) \cdot \mathbf{n}=\mathrm{G}_{\ell} \text { on } \partial \mathbf{H}_{\infty}
\end{array}\right.
$$

When dealing with several perturbations, the evaluation of the solution at first order requires to take into account the potential interaction between perturbations. It has been proven in (Bonnaillie-Noël et al., 2009) that if the distance between the centers of two neighbor perturbations is written as $\varepsilon^{\alpha}$ with $0<\alpha<\frac{2}{3}$, the perturbations can 
be considered as far and no interaction needs to be taken into account at first order. The solution is then approximated by:

$$
\mathbf{u}_{\varepsilon}(\mathbf{x}) \simeq \mathbf{u}_{0}(\mathbf{x})-\sum_{i=1}^{\text {nb defects }} \varepsilon_{i}\left[\boldsymbol{\alpha}_{1}^{i} \mathrm{~V}_{1}^{i}\left(\frac{\mathbf{x}}{\varepsilon_{i}}\right)+\boldsymbol{\alpha}_{2}^{i} \mathrm{~V}_{2}^{i}\left(\frac{\mathbf{x}}{\varepsilon_{i}}\right)\right]
$$

\section{Failure description}

The description of the failure of a structure presenting geometrical perturbations is decomposed into two phases:

- in a first step, the stress concentration due to the presence of micro-defects is evaluated with the help of asymtptotic analysis,

- in a second step, we describe the localization zones and cracks developing from the stress concentration zones through a strong discontinuity model.

Numerically, the two previous steps are performed considering a coarse description of the geometry: only a discretization of the unperturbed domain is considered.

For structures presenting several defects, depending on the loading and on the geometry, it can be necessary to deal simultaneously with asymptotic analysis in some parts of the structure still in the elastic regime and with damage development in some other parts where cracks yet initiated. To that purpose, a dedicated tool incorporating both aspects previously described is to be developed.

\subsection{Kinematic enrichment for the asymptotic analysis}

Taking into account the asymptotic fields in a numerical tool can be achieved by the use of the partition of unity method (Melenk et al., 1996) leading to the evaluation of the influence of perturbations while using a coarse discretization of the unperturbed domain. The standard variational Finite Element space is enriched by the approximation $\tilde{\mathbf{V}}_{\ell}$ of the profiles $\mathbf{V}_{\ell}$. The approximations $\tilde{\mathbf{V}}_{\ell}$ are computed on a truncated domain $\mathbf{H}_{R}=\mathbf{H}_{\infty} \cap B(\mathbf{0}, R)$ where $R$ is chosen as large as possible. We bring the vanishing condition at infinity on the artificial boundary $\partial \mathbf{H}_{R} \backslash \partial \mathbf{H}_{\infty}$.

As the profiles decay at infinity, their influence is very local and thus, only a neighborhood of the perturbations needs to be enriched. The displacement field, as suggested by [4], is then given by:

$$
\mathbf{u}_{\varepsilon}^{h}(\mathbf{x})=\mathbf{u}_{0}^{h}(\mathbf{x})-\sum_{i=1}^{\text {nb defects }} \varepsilon_{i} \sum_{\ell=1}^{2} \sum_{j \in \mathcal{J}_{i}} N^{j}(\mathbf{x})\left[\boldsymbol{\alpha}_{j \ell, 1}^{i} \tilde{\mathrm{V}}_{\ell, 1}^{i}\left(\frac{\mathbf{x}}{\varepsilon_{i}}\right)+\boldsymbol{\alpha}_{j \ell, 2}^{i} \tilde{\mathrm{V}}_{\ell, 2}^{i}\left(\frac{\mathbf{x}}{\varepsilon_{i}}\right)\right]
$$

where $\mathcal{J}_{i}$ denotes the indices of the nodes located in the enrichment zone related to perturbation $i . N^{j}$ are the standard shape functions associated to node $j$ and $\boldsymbol{\alpha}_{j \ell, k}^{i}$ is a 
two-component vector consisting of the degrees of freedom related to the enrichment function $\tilde{\mathrm{V}}_{\ell, k}^{i}$.

The computation is performed on the discrete unperturbed domain. The presence of the perturbations and their geometry are taken into account through a dedicated numerical integration based on the exact geometry.

Concerning the numerical computation of the $\boldsymbol{\alpha}_{j \ell, k}^{i}$, as observed in the context of XFEM (Chahine et al., 2007), if all those enriched degrees of freedom are kept free in the enrichement areas, the problem to be solved is badly conditioned. In order to circumvent this difficulty, we impose the following equalities suggested by the asymptotic analysis:

$$
\left(\alpha_{j \ell, 1}^{i}\right)_{1}=\left(\alpha_{j \ell, 2}^{i}\right)_{2} \quad \text { and } \quad\left(\alpha_{j \ell, 1}^{i}\right)_{2}=\left(\alpha_{j \ell, 2}^{i}\right)_{1} .
$$

Those equalities are ensured by appealing to a master/slave strategy leading to the resolution of an augmented problem:

$$
\left[\begin{array}{ccc}
\mathbf{K}_{\mathbf{u u}}^{0} & \mathbf{0} & \mathbf{0} \\
\mathbf{K}_{\boldsymbol{\alpha u}}^{\varepsilon} & \mathbf{K}_{\boldsymbol{\alpha} \boldsymbol{\alpha}}^{\varepsilon} & \boldsymbol{\Pi}^{\top} \\
\mathbf{0} & \mathbf{\Pi} & \mathbf{0}
\end{array}\right]\left[\begin{array}{c}
\mathbf{u}_{0} \\
\boldsymbol{\alpha} \\
\boldsymbol{\lambda}
\end{array}\right]=\left[\begin{array}{c}
\mathbf{f}_{0} \\
\mathbf{f}_{\boldsymbol{\alpha}} \\
\mathbf{0}
\end{array}\right]
$$

where $\mathbf{K}_{\mathbf{u u}}^{0}$ is the standard stiffness matrix computed on the unperturbed domain, $\Pi$ denotes the projection operator over the equality constraints [6], $\mathbf{K}_{\alpha \boldsymbol{\alpha}}^{\varepsilon}$ and $\mathbf{K}_{\boldsymbol{\alpha} \mathbf{u}}^{\varepsilon}$ denote respectively the part of the total stiffness associated to the added degrees of freedom and the coupled part. The vector $\boldsymbol{\lambda}$ is the Lagrange multiplier associated with the constraints [6] and finally, $\mathbf{f}_{0}$ and $\mathbf{f}_{\boldsymbol{\alpha}}$ denote the external loading.

The two matrices $\mathbf{K}_{\boldsymbol{\alpha} \boldsymbol{\alpha}}^{\varepsilon}$ and $\mathbf{K}_{\boldsymbol{\alpha} \mathbf{u}}^{\varepsilon}$ are computed by using a dedicated integration strategy:

- a partitioning, typically obtained by meshing, of the elements affected by the perturbation is carried out in order to compute all the quantities involving the profiles,

- in the vicinity of the perturbation, the order of integration is also increased in order to capture the evolution of profiles.

\subsection{Field transfer: coupling of asymptotic analysis and strong discontinuity approach}

The stress concentrations generated by the presence of micro-defects in hand, one can continue the computation by using the strong dicontinuity approach (SDA) (see (Simo et al., 1993; Oliver, 1995; Brancherie et al., 2009)) in order to track the developement of high damage zones initiated on the geometrical perturbations.

For that purpose, it is necessary to project the field obtained from the asymptotic analysis to the variational space used for strong discontitnuity approach. Indeed the kinematic enrichments of the variational spaces associated to the asymptotic analysis and the strong discontinuity approach are not compatible. The strategy proposed 
herein is to design a field transfer ensuring the transfer of the displacement field from one variational space to the other one. Let's denote $\mathcal{V}_{S D A}$ the variational space associated to the SDA, the displacement field used as initial value for the continuation of the computation is obtained as the solution of a minimisation problem given as:

$$
\min _{\mathbf{u} \in \mathcal{V}_{S D A}} J(\mathbf{u})=\mathcal{E}\left(\mathbf{u}-\mathbf{u}_{\varepsilon}^{h}\right) \quad \text { u.c. } \quad \mathbf{u}=\mathbf{u}^{d} \text { on } \Gamma_{d}
$$

where $\mathcal{E}(\mathbf{v})=\frac{1}{2} \int_{\Omega_{\varepsilon}} \boldsymbol{\sigma}(\mathbf{v}): \varepsilon(\mathbf{v}) d \Omega$. The proposed strategy is then based on the construction of a displacement field in $\mathcal{V}_{S D A}$ producing a strain energy being as close as possible to the strain energy produced by the solution obtained on the perturbed domain $\mathbf{u}_{\varepsilon}^{h}$. The solution of such a minimisation problem under constraint is obtained as the solution of a linear set of equations.

This projected displacement field $\mathbf{u}$ is then used to compute the corresponding stress introduced as an initial value for the continuation of the computation with strong discontinuity approach.

\section{Numerical results}

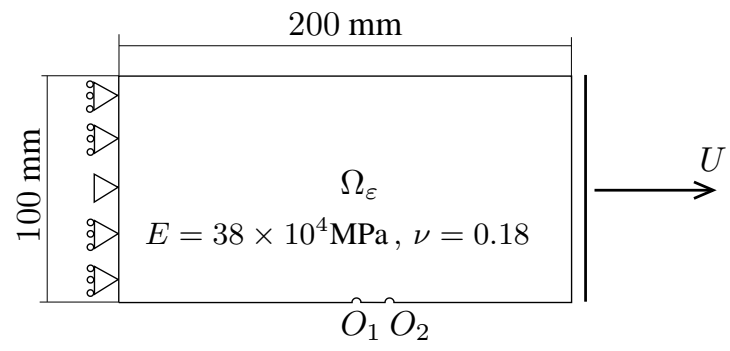

(a) Problem definition: geometry, loading and material properties

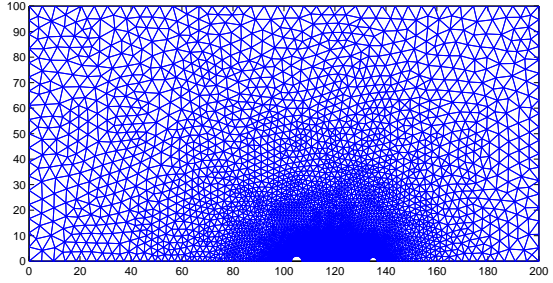

(b) Fine discretization of the domain (reference computation)

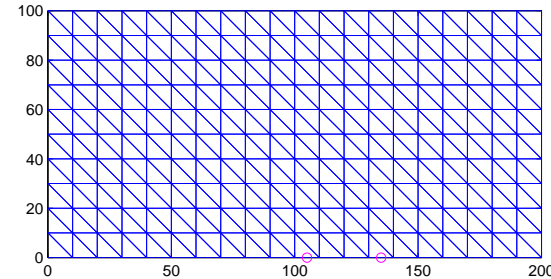

(c) Coarse mesh for computation of $\mathbf{u}_{\varepsilon}^{h}$

Figure 2. Problem definition: geometry, loading, material properties and discretizations 
We present here the results obtained considering a domain submitted to a tensile load (Figure 2(a)). The domain is pierced by two perturbations: the first one is centered at point $O_{1}=(105,0)$ and is of radius $2 \mathrm{~mm}$ and the second of radius $1.5 \mathrm{~mm}$ is centered at point $O_{2}=(135,0)$.

In order to validate the enrichment strategy adopted for the description of the influence of small defects, we compare the results obtained in terms of displacement and stress fields for, a so called, reference computation carried out on a fine discretization of the real geometry (Figure 2(b)) by standard Finite Element approach, and an enriched computation performed on a coarse discretization (Figure 2(c)) of the unperturbed domain.

Figure 3 gives the obtained results in terms of the displacement field in the direction of the traction for both discretization and interpolations. The relative error between those two computations is lower than $0.25 \%$ allowing to conclude that the proposed strategy gives satisfactory results regarding the one provided by the asymptotic analysis.

Figure 4 gives the stress field $\sigma_{x x}$ obtained form the standard reference computation and the enriched one. Figure 4(c) illustrates the relative error computed in terms of strain energy. For the considered case, the relative error integrated over the whole domain is less than $0.01 \%$, it is very concentrated at the very small vicinity of the perturbations on the boundary of the domain where the strain energy is about zero (leading to high relative error). Those results confirm that, for relatively close perturbations, the profiles computed from the asymptotic analysis at first order are sufficient to obtain a good approximation of the solution, no interactions between the inclusions need to be taken into account.

From the displacement obtained through the enriched computation, the field transfer presented in Section 3.2 is performed as soon as the maximal principal stress reaches the limit value chosen for the initiation of cracks. Figure 5(a) represents the stress field obtained from the rebuilt displacement after transfer. This field is the one introduced as initial value for the continuation of the computation with strong discontinuity approach. We can observe that the stress concentration due to the presence of the perturbations are well reproduced, crack initiation takes place at the right location. Figure 5(b) gives the orientation and opening of the introduced discontinuities at the end of the loading process.

We can see that the crack leading to complete failure of the domain initiated on the bigger perturbation centered on point $O_{1}$. Indeed, the development of this crack is accompanied with elastic unloading of the rest of the domain preventing the development of a second crack. 
172 EJCM - 19/2010. Giens 2009

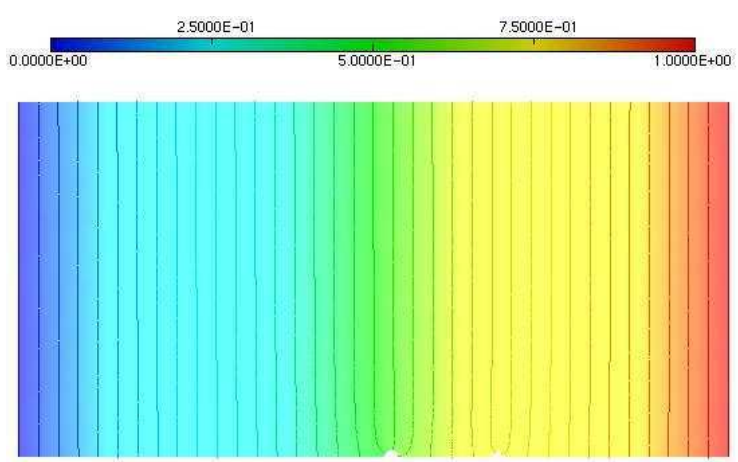

(a) Reference computation on the fine discretization

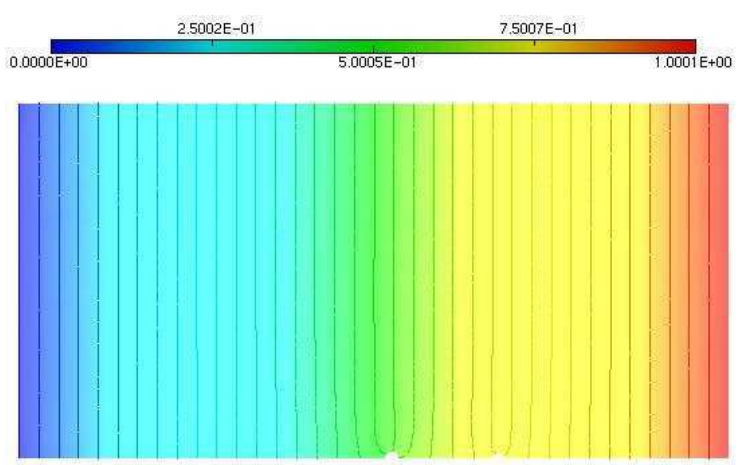

(b) Computation with kinematic enrichment on the coarse discretization

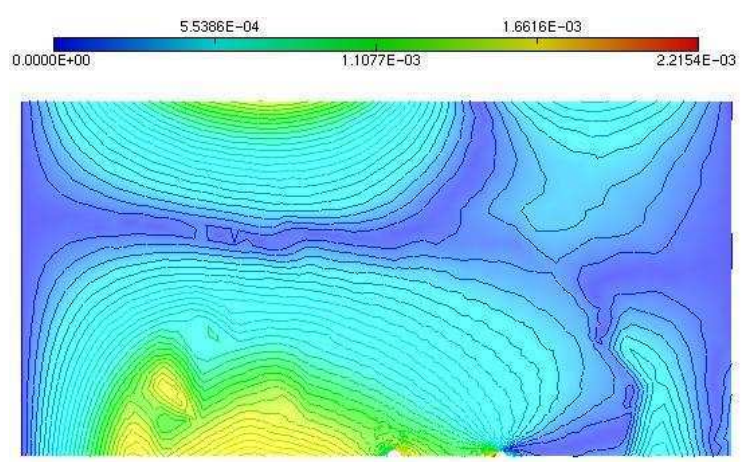

(c) Relative error

Figure 3. Displacement field $u_{x}$ obtained by standard and enriched computation, relative error map 


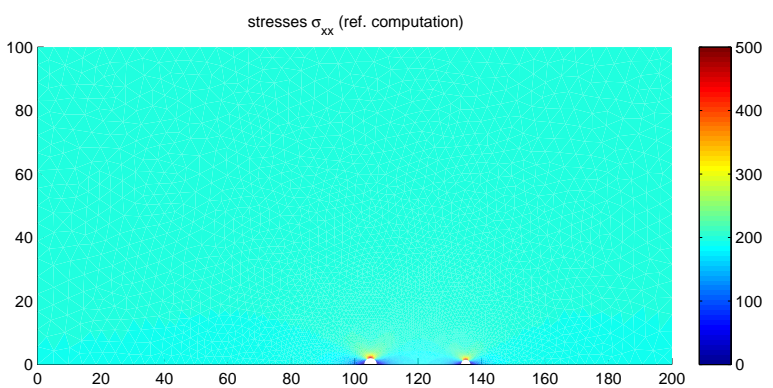

(a) Reference computation

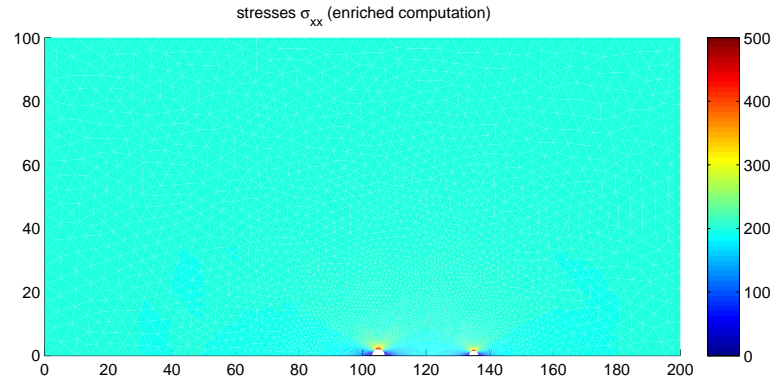

(b) Computation with kinematic enrichment

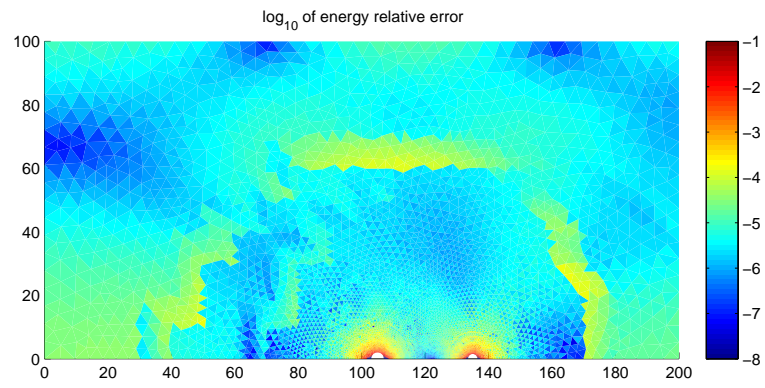

(c) Relative error (strain energy)

Figure 4. Stress field $\sigma_{x x}$ obtained by standard and enriched computation, relative strain energy error map 


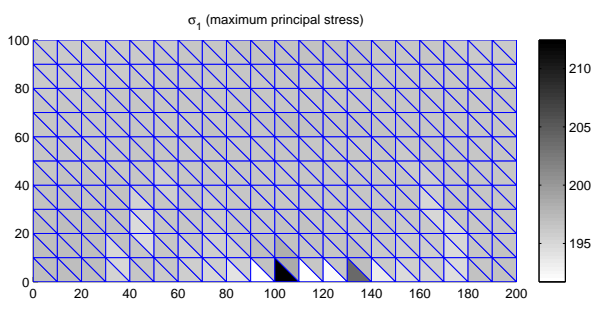

(a) Reconstructed principal maximum stress

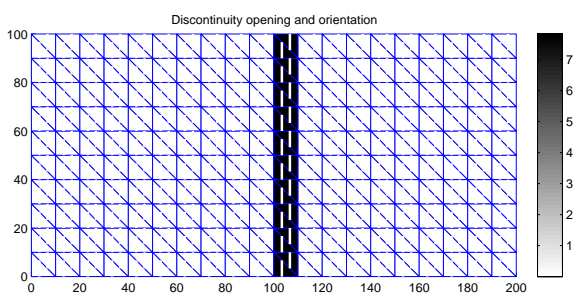

(b) Discontinuities orientation and crack opening (mm)

Figure 5. Difference between the reconstructed displacement field after transfer and the field obtained on the unperturbed domain, reconstructed maximal principal stress

\section{Conclusion}

We have presented a strategy allowing to take into account the influence of microdefects on the behavior till rupture of structures. The key point of the proposed approach is that this description is achieved without any fine description of the exact geometry of the domain but rather with a coarse description of the unperturbed domain, the perturbations being incorporated in the computation through a kinematic enrichment of standard Finite Element method.

This enrichment is provided by a multi-scale asymptotic analysis of Navier equations for linear elasticity. The description of the initiation and developement of localization zones leading to the apparition of cracks is ensured by the use of a strong discontinuity approach. A field transfer operator has been designed in order to couple those two approaches during all the loading process. 


\section{References}

Bonnaillie-Noël V., Dambrine M., Tordeux S., Vial G., "Interactions between moderately close inclusions for the Laplace equation", Math. Models Methods Appl. Sci., vol. 19, n 10, p. 1853-1882, 2009.

Brancherie D., Ibrahimbegović A., "Novel anisotropic continuum-discrete damage model capable of representing localized failure of massive structures. Part I: theoretical formulation and numerical implementation", Engineering Computations, vol. 26, n ${ }^{\circ} 1-2$, p. 100-127, 2009.

Chahine E., Laborde P., Pommier J., Renard Y., Salaun M., "Study of some optimal xfem type methods", Computational Methods in Applied Sciences, vol. 5, p. 27-40, 2007.

Dambrine M., Vial G., "Influence of a boundary perforation on the Dirichlet energy", Control and Cybernetics, vol. 34, $\mathrm{n}^{\circ}$ 1, p. 117-136, 2005.

Melenk J.M., Babuška I., "The partition of unity finite element method: Basic theory and applications", Computer Methods in Applied Mechanics and Engineering, vol. 136, p. 289-314, 1996.

Oliver J., "Continuum Modelling of Strong Discontinuities in Solid Mechanics", in D. Owen, E. Oñate, E. Hinton (eds), Computational plasticity IV. Fundamentals and Applications, Barcelone, p. 455-480, 1995.

Simo J.C., Oliver J., Armero F., "An Analysis of Strong Discontinuity Induced by Strain Softening Solutions in Rate-Independent Solids", Journal of Computational Mechanics, vol. 12, p. $277-296,1993$. 
